\title{
Norois
}

Environnement, aménagement, société

\section{Dater les anciennes pêcheries par les niveaux marins approche méthodologique et perspectives géoarchéologiques : le Bas Léon, nord Finistère, Bretagne}

Geoarchaeology of the ancient fish-traps and sea level changes: cross approaches on coastal questions

Marie-Yvane Daire et Loïc Langouët

\section{(2) OpenEdition}

\section{Journals}

Édition électronique

URL : https://journals.openedition.org/norois/3680

DOI : 10.4000/norois.3680

ISSN : 1760-8546

\section{Éditeur}

Presses universitaires de Rennes

\section{Édition imprimée}

Date de publication : 30 novembre 2011

Pagination : 69-93

ISBN : 978-2-7535-1765-3

ISSN : 0029-182X

\section{Référence électronique}

Marie-Yvane Daire et Loïc Langouët, « Dater les anciennes pêcheries par les niveaux marins approche méthodologique et perspectives géoarchéologiques : le Bas Léon, nord Finistère, Bretagne », Norois [En ligne], 220 | 2011, mis en ligne le 30 novembre 2013, consulté le 14 janvier 2022. URL : http:// journals.openedition.org/norois/3680 ; DOI : https://doi.org/10.4000/norois.3680

Ce document a été généré automatiquement le 14 janvier 2022.

(c) Tous droits réservés 


\section{Dater les anciennes pêcheries par les niveaux marins approche méthodologique et perspectives géoarchéologiques : le Bas Léon, nord Finistère, Bretagne}

Geoarchaeology of the ancient fish-traps and sea level changes: cross approaches on coastal questions

Marie-Yvane Daire et Loïc Langouët

1 Les anciennes pêcheries sont aujourd'hui, à juste titre, considérées comme des vestiges archéologiques susceptibles d'éclairer les modalités d'exploitation des ressources et les interactions homme-milieu des sociétés du passé. Par son caractère très récent, cette archéologie des pêcheries de Bretagne requiert encore la construction de référentiels chrono-culturels et environnementaux.

2 Les recherches menées à travers l'Europe montrent que les premiers pièges à poissons remontent au Mésolithique ; c'est le cas des pêcheries en bois rencontrées sur les côtes de la Mer Baltique, notamment au Danemark en Allemagne (Pedersen, 1995). Elles se développent ensuite au cours du Néolithique (Fisher, 2007), en Allemagne (Lübke, 2007), en Hollande (Maarleveld, 1993), en Grande-Bretagne et en Irlande, (Bell et al., 2000 ; Cooper, 2005; O'Sullivan, 1994, 2001 et 2003, Bell, 2007). La chronologie des premières pêcheries en bois a évolué récemment avec la découverte des 5 pêcheries mésolithiques en noisetier de la rivière Liffey (Dublin, Irlande), implantées à $-6,3 \mathrm{~m}$ sous le niveau des plus basses mers et construites vers $6100-5700$ av. J.-C. (McQuade et O'Donnell, 2007). Tout comme en Europe continentale, ces pêcheries précoces impliquent l'existence de communautés organisées qui savent attraper des poissons en utilisant les flux de marées, qui savent aussi élaborer des clayonnages et tisser des paniers. Géographiquement plus près de nous, dans le secteur de Saint-Jean-Le-Thomas 
(Manche) les recherches récentes ont révélé l'existence de deux pêcheries remontant à l'Âge du Bronze ancien (environ -2000 av. J.-C.) (Billard et al., 2006)ํ․

Qu'ils soient en bois (bouchots) ou en pierres (parcs ou écluses), les barrages à poissons que nous retrouvons généralement dans la partie basse de la bande intertidale, voire dans le domaine infratidal, répondent à des principes généraux assez ubiquistes dans le temps et dans l'espace (fig. 1). Il s'agit en effet de pièges construits en bois ou en pierre, destinés à capturer poissons et crustacés par filtrages des flux hydrodynamiques maritimes et fluviaux liés aux marées ou aux courants. De formes et dimensions variées, ces pièges ont été utilisés jusqu'à nos jours dans certains secteurs géographiques comme la baie du Mont-Saint-Michel.

\section{Le repérage des pêcheries}

En 2007, l'inventaire initial (environ 150 barrages à poissons) reposait essentiellement sur des dépouillements d'archives ou de bibliographies. Depuis, la recherche méthodologique des anciennes pêcheries a été réalisée principalement à partir des couvertures photographiques aériennes de l'IGN et de photographies obliques à très basse altitude, disponibles sur Internet. Les anciennes photographies en noir et blanc de l'IGN (1952, 1961, 1977), disponibles gratuitement sur Internet, ont été systématiquement dépouillées. Une étude complémentaire d'autres couvertures IGN (entre 1949 et 1998) a été réalisée au Laboratoire Costel. Par ailleurs le site GeoLittoral qui diffuse les photographies Ortho Littoral 2000 et 2006, prises lors de marées relativement basses, offre la possibilité d'y mesurer avec précision les longueurs des barrages. Une base de données $\mathrm{PEB}^{2}$ regroupe tous les renseignements recueillis.

Figure 1 : Pièges à poissons sur estran : bouchot et parc ou écluse/Fish-traps on the intertidal belt: wooden trap and dam or weir

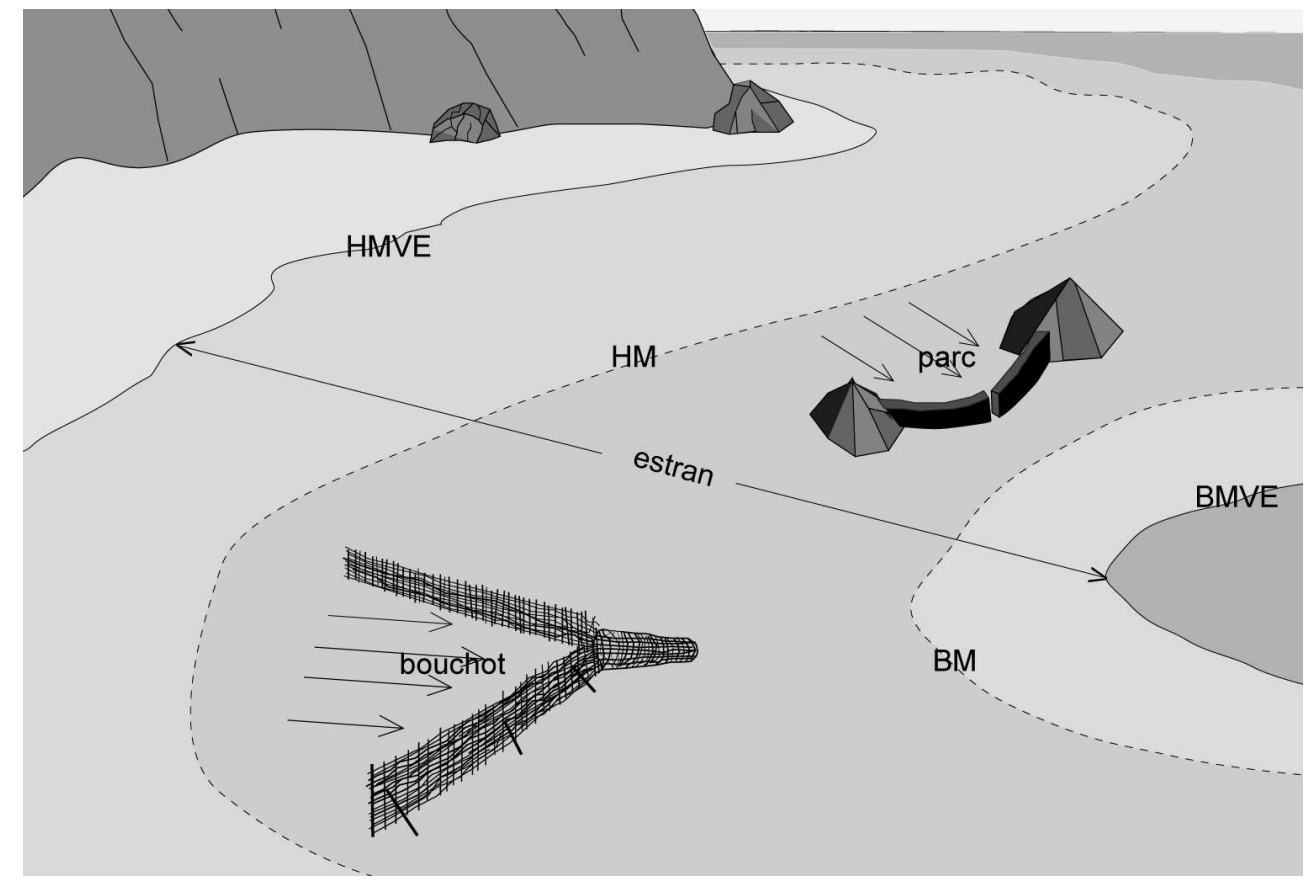

Il est aisé de reconnaître les barrages de pêcherie et de les distinguer d'affleurement rocheux ou de lignes d'algues de laisse. Les barrages empierrés sur fond sableux, 
généralement clairs, sont les plus faciles à repérer, car les algues brunes qui se sont ancrées sur les pierres améliorent le contraste; par contre les empierrements qui reposent sur un fond rocheux sont plus difficiles à identifier; ils sont généralement repérés lors de visites de l'estran. Un piège à poissons n'a été pris en compte dans la base PEB que s'il est identifiable au minimum sur deux vues de missions différentes. Les vérifications au sol qui demandent du temps (720 pièges à ce jour en Bretagne) permettent d'avoir des renseignements complémentaires sur l'architecture, certaines dimensions (largeur et indirectement hauteur), le niveau de la base noté $\mathrm{N}_{b}$, etc. Dans un premier temps, le niveau de la base d'un barrage $\mathrm{N}_{\mathrm{b}}$ est évalué à partir des indications altimétriques des cartes SHOM et IGN. Dans un second temps, ce niveau est affiné sur place en mesurant la dénivellation entre le niveau de la mer, tel jour à telle heure, et la base du barrage (ou le niveau du pertuis). À l'aide des données SHOM et en tenant compte de la pression atmosphérique et du vent, on obtient une valeur plus précise de $\mathrm{N}_{\mathrm{b}}$ repéré par rapport au zéro SHOM (Langouët et Daire, 2010). En Bretagne, la caractérisation systématique de ces anciens barrages permet de dégager un certain nombre de critères ou de règles qui, en attendant des recherches de terrain plus approfondies (sondages, fouilles), constituent une première étape dans la contextualisation de ces vestiges : il s'agit en effet de proposer, à terme, une approche globale des occupations humaines littorales, intégrant les données de l'archéologie terrestre, intertidale et subaquatique. Mais, pour établir un lien entre les sites terrestres connus et identifiés pour certaines périodes (habitats, notamment) et les barrages à poissons, le jalon chronologique est incontournable. Or, les vestiges de pêcheries encore visibles de nos jours sont en très grande majorité en pierres, du fait de la mauvaise conservation des bois. Les parcs (ou écluses) en pierres sont difficiles à dater actuellement par des méthodes scientifiques dites objectives, du moins en l'absence d'investigations plus approfondies (sondages, fouilles, etc.); par ailleurs, la quantité de données à traiter sur plusieurs centaines de vestiges nous a incités à rechercher des principes (règles de corrélations) en vue de traitements statistiques permettant d'avancer, par touches successives, dans les domaines de la chronologie de ces barrages de pêcheries et dans leur implantation par rapport aux niveaux marins dont on sait qu'ils ont varié au fil des siècles.

6 Le but de notre étude actuelle a été de tester ces règles de corrélation entre le niveau topographique d'implantation d'un barrage et le niveau marin dans la région du BasLéon (Finistère) où l'on dispose de repères pour la variation relative du niveau marin. Les apports chronologiques et les perspectives géoarchéologiques d'une telle approche croisée seront ensuite dégagés.

\section{Règles de fonctionnement}

7 Si l'on devait aménager une pêcherie aujourd'hui, certaines règles d'implantation seraient à respecter : l'une concerne la nécessaire accessibilité de la zone d'installation du barrage, au regard des marées, accessibilité indispensable pour sa construction, son entretien et son utilisation; l'autre règle concerne l'accessibilité du biez du point de vue de la ressource halieutique transportée par la mer, et débouche sur l'efficacité du piégeage et des prises. Cette double accessibilité, à partir de la terre pour l'Homme et à partir de la mer pour la ressource, est liée à la position du barrage par rapport à diverses contraintes topographiques et hydrodynamiques. 
Pour comprendre les processus, il faut préalablement définir certaines caractéristiques physiques d'une pêcherie et rappeler la terminologie qui lui est associée (fig. 2). Un barrage de pêcherie comprend, en règle générale, un ou deux bras, continus ou segmentés appelés "pannes»; une ou plusieurs ouvertures désignées ici comme "pertuis » peuvent avoir été aménagées dans le barrage pour y placer un filet ou une nasse destinée à recueillir les prises. Enfin, en arrière du barrage, le «bassin » où sont piégés poissons et crustacés est dénommé "biez». Dans le cas des pêcheries dépourvues d'ouverture, les prises s'effectuent dans le biez au moyen de filets ou de haveneaux. Le barrage repose sur l'estran ou dépasse de celui-ci à un niveau $\mathrm{N}_{\mathrm{b}}$ (niveau de la base) ; il est souvent le même que celui de l'ouverture ou pertuis. Ce barrage ayant une certaine hauteur $\mathrm{h}$, sa partie haute est à un niveau $\mathrm{N}_{\mathrm{h}}$; celui-ci, combiné au plan et dimensions fournis par les pannes, définit le biez à la fois en surface et volume.

\section{Règle régissant l'accessibilité anthropique}

En morte-eau extrême (coefficient 20), HMME et BMME caractérisent les niveaux théoriques de haute et basse mers tandis que, en vive-eau extrême (coefficient 120), HMVE et BMVE sont les niveaux théoriques de haute et basse mers. Le niveau BMVE a une autre signification importante: c'est le niveau zéro des cartes marines ou le 0 SHOM (Service Hydrographique et Océanographique de la Marine) à partir duquel tous les niveaux précités sont calculés. Le niveau HMVE, mesuré ainsi par rapport au 0 $\mathrm{SHOM}$, définit le marnage maximal local MM. Le niveau moyen $\mathrm{N}_{\mathrm{m}}$ est la moitié du marnage maximal. À partir des annuaires de marées, il est très facile de vérifier que, quel que soit le port breton, il existe une relation statistique reliant le niveau BMME au marnage maximal MM (Langouët et al., 2008; Daire et Langouët, 2010), avec une précision relative de $1 \%$ :

Figure 2 : Les principales caractéristiques d'un barrage à poissons, lors d'une marée quelconque/ Main characteristics of a fish-trap during any tide

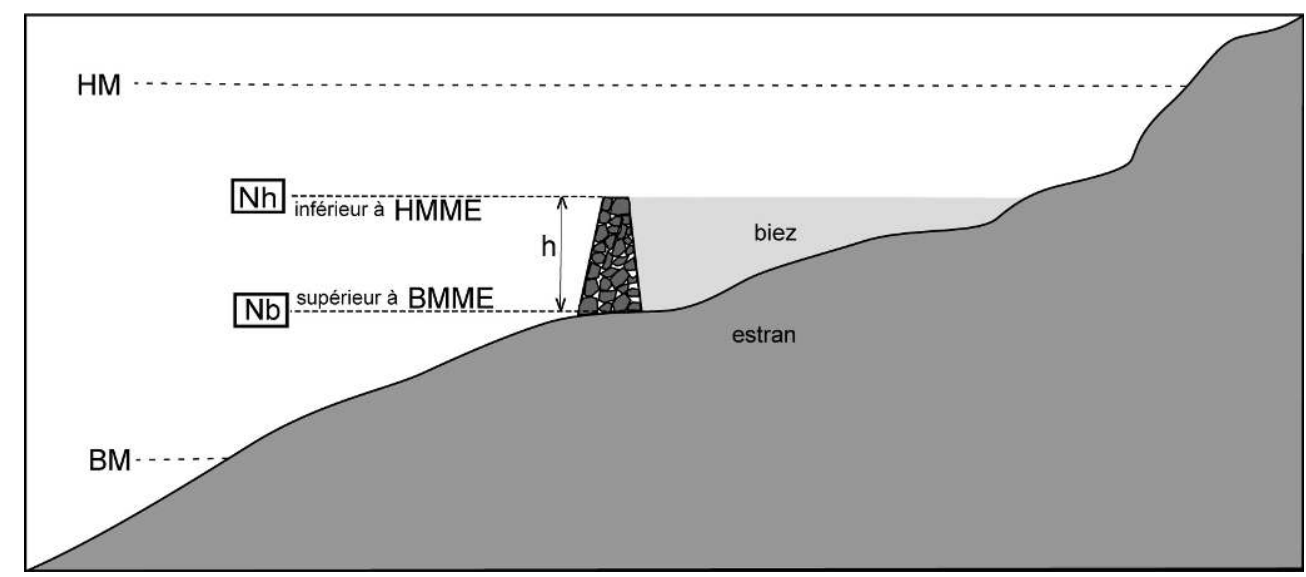

11 Pour avoir accès à la base d'un barrage, du fait que tout niveau de basse mer BM est inférieur à BMME, il est nécessaire que :

$12 \mathrm{~N}_{\mathrm{b}} \geq 0,416_{*} \mathrm{MM}$ 


\section{Règle regissant l'accessibilité par la ressource}

\section{Les barrages à poissons de la côte sud}

21 $(5,85 \mathrm{~m}$ à Penmarc' $\mathrm{h}, 5,63 \mathrm{~m}$ à Concarneau, 6,10 $\mathrm{m}$ à La Trinité-sur-Mer et Pénestin, etc.). La dernière condition définit, pour cette zone géographique, la hauteur maximale d'un barrage "pleinement opérationnel » autour de $1 \mathrm{~m}$. Avec une telle valeur de $\mathrm{h}$, les deux conditions d'accessibilité se résument à une seule et imposent que $\mathrm{N}_{\mathrm{b}}$ soit au niveau BMME (précision finale de $7 \%$ ).

\section{Les barrages à poissons des côtes ouest et nord}

Pour les côtes bretonnes comprises entre Douarnenez ( $\mathrm{MM}=7,32 \mathrm{~m}$ ) et le Mont-SaintMichel $(\mathrm{MM}=14,40 \mathrm{~m})$, les deux règles d'accessibilité d'une pêcherie ne peuvent pas bénéficier de cette simplification du fait de marnages plus importants que sur la côte sud. La double inégalité précitée débouche sur une latitude brute d'implantation de $\mathrm{N}_{\mathrm{b}}$ pouvant atteindre 1,40 $\mathrm{m}$ dans l'hypothèse d'un barrage haut de $1 \mathrm{~m}$ (Daire et Langouët, 2010) ; la précision finale du niveau d'implantation dans l'estran mesuré par rapport au 0 SHOM est de l'ordre de $20 \%$. C'est lors de ces implantations dans l'estran que peuvent intervenir des choix et des contraintes. Il serait logique de retenir une implantation dans la partie la plus haute de l'estran pour des questions de rapidité d'accès et des diminutions de déplacement. Mais on ne saurait oublier que la nature des prises peut dépendre du niveau du barrage dans l'estran; ce ne sont pas les mêmes espèces qui peuvent être piégées, quel que soit le niveau de la base du barrage. Un autre facteur peut aussi intervenir: celui des contraintes topographiques. Dans la majorité des installations, les barrages en pierres s'appuient sur des têtes rocheuses entre lesquelles un courant de jusant se produit régulièrement; ces émergences rocheuses peuvent avoir influencé l'implantation d'un barrage au sein de la latitude évoquée plus haut. 
Pour les côtes ouest et nord de la Bretagne, aucune règle ne permet de préciser systématiquement le niveau qui sera choisi dans une bande de niveaux définie par les conditions exposées supra.

\section{Barrages de pêcheries et niveaux marins}

23 On vient d'exposer les conditions d'installation d'un barrage de pêcherie pour que la collecte de poissons et crustacés puisse se faire lors des 700 marées qui se produisent par an dans les conditions actuelles ${ }^{3}$. Pour des périodes historiques récentes, ces règles semblent avoir été respectées. Ainsi, pour les $\mathrm{XVI}^{\mathrm{e}}$ et $\mathrm{XVII}{ }^{\mathrm{e}}$ siècles, les cartes des Ingénieurs-Géographes ${ }^{4}$ indiquent que les pertuis de la cinquantaine de pêcheries de la baie de Cancale sont situés sur la ligne de "la laisse de basse mer de morte-eau ", soit au niveau BMME. Par ailleurs, entre le $\mathrm{VI}^{\mathrm{e}}$ et le $\mathrm{xV}^{\mathrm{e}}$ siècle, le respect de cette même règle d'implantation de barrages monastiques insulaires a permis de déterminer une montée relative du niveau marin de $0,90 \pm 0,1 \mathrm{~m}$ en 1000 ans (Langouët et al., 2008). On peut donc partir du postulat que ces mêmes règles d'implantation ont été suivies dans les temps plus anciens puisqu'elles reposent sur une optimisation des choix techniques et des investissements énergétiques.

En théorie, pour n'importe quel barrage de Bretagne, le nombre de marées "pêchantes ", c'est-à-dire rendant ce barrage accessible bi-quotidiennement, varie entre 600 et 0 . Dans le secteur du Bas-Léon, pour 18 des pièges, le pourcentage des marées " pêchantes » est relativement faible, voire nul (tableau 1, colonne Nb marées). La faible efficacité actuelle de ces pêcheries ne peut alors s'expliquer qu'à la lumière du phénomène de montée du niveau marin, ce qui induit une datation plus ou moins ancienne de ces structures. Nous verrons infra que dans l'analyse d'une corrélation entre l'implantation des barrages et le positionnement des niveaux marins correspondant, l'une des données incontournables est la hauteur initiale des barrages puisqu'elle conditionne l'accès des proies.

Tableau 1: Quelques caractéristiques des pièges à poissons de la côte du Bas-Léon/Main features of the fish traps alongside the coasts of Bas-Léon 


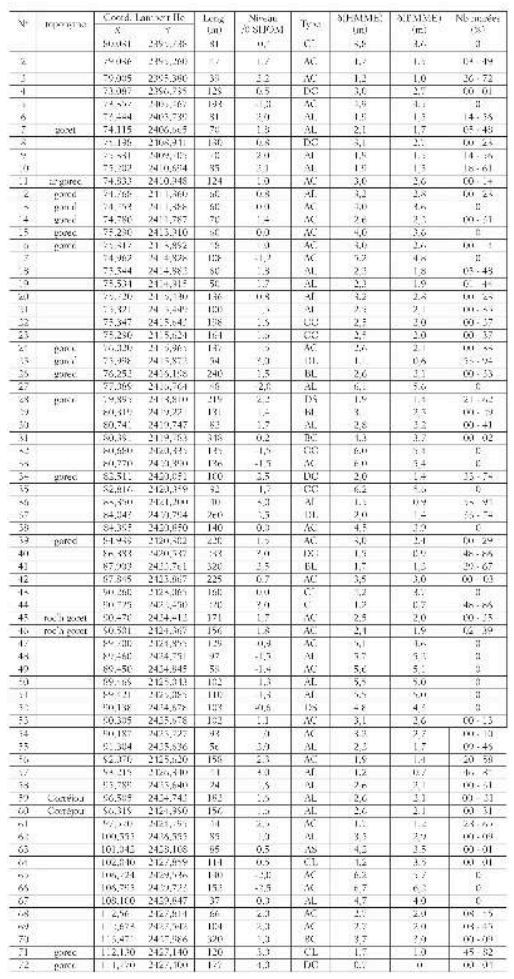

\section{La hauteur des barrages}

La hauteur des barrages en bois est difficile à restituer du fait de la dégradation, voire la disparition, des éléments ligneux en superstructures. Par contre, dans le cas des anciens pièges en pierres, certains types d'architecture nous fournissent l'opportunité d'une évaluation métrique, notamment de la hauteur initiale de la structure : soit par les dimensions des pierres dressées ayant constitué leurs parements (indication de hauteur minimale), soit par une reconstitution de la coupe du barrage à partir de la largeur de sa base et de la section des pierres étalées (fig. 3 ; Daire et Langouët, 2010 ; Langouët et Daire, 2010).

Ainsi les hauteurs initiales ont pu être évaluées pour une bonne vingtaine de barrages des côtes bretonnes, fournissant une hauteur moyenne de 0,9 $\pm 0,3 \mathrm{~m}$ dans l'hypothèse de barrages aux parois verticales; il s'agit, à l'évidence, d'une hauteur minimale pour plusieurs raisons: un éventuel phénomène d'ensablement, une érosion des superstructures, une possible récupération de pierres et une section trapézoïdale dont le fruit des parements est difficile à évaluer. Des indications de hauteurs sont également fournies par la documentation historique. Ainsi plusieurs arrêts et les ordonnances $d u \mathrm{XVII}^{\mathrm{e}}$ au $\mathrm{XIX}^{\mathrm{e}}$ siècle limitaient la hauteur des barrages en pierres à 4 pieds, soit 1,30 $\mathrm{m}$. Cette valeur maximale correspond, entre autres, à une réalité, pleine de sagesse, basée sur l'observation de phénomènes naturels, celle de permettre à de modestes barrages de résister aux courants et à la houle des côtes bretonnes.

La synthèse des données historiques précitées $(\max 1,30 \mathrm{~m})$ et des valeurs archéologiques retrouvées pour une vingtaine de barrages ( $\min 0,9 \pm 0,3 \mathrm{~m}$ ) conduit à proposer comme une hypothèse de travail basée sur une hauteur moyenne des anciens barrages de pêcheries de Bretagne de 1 $\pm 0,2 \mathrm{~m}$. 


\section{Pêcheries et variations relatives des niveaux marins}

Figure 3 : Évaluation de la hauteur minimale initiale $h$ d'un ancien barrage à deux parements verticaux/Evaluation of the minimum initial hight $h$ for an old stone fishweir caracterized by two vertical faces

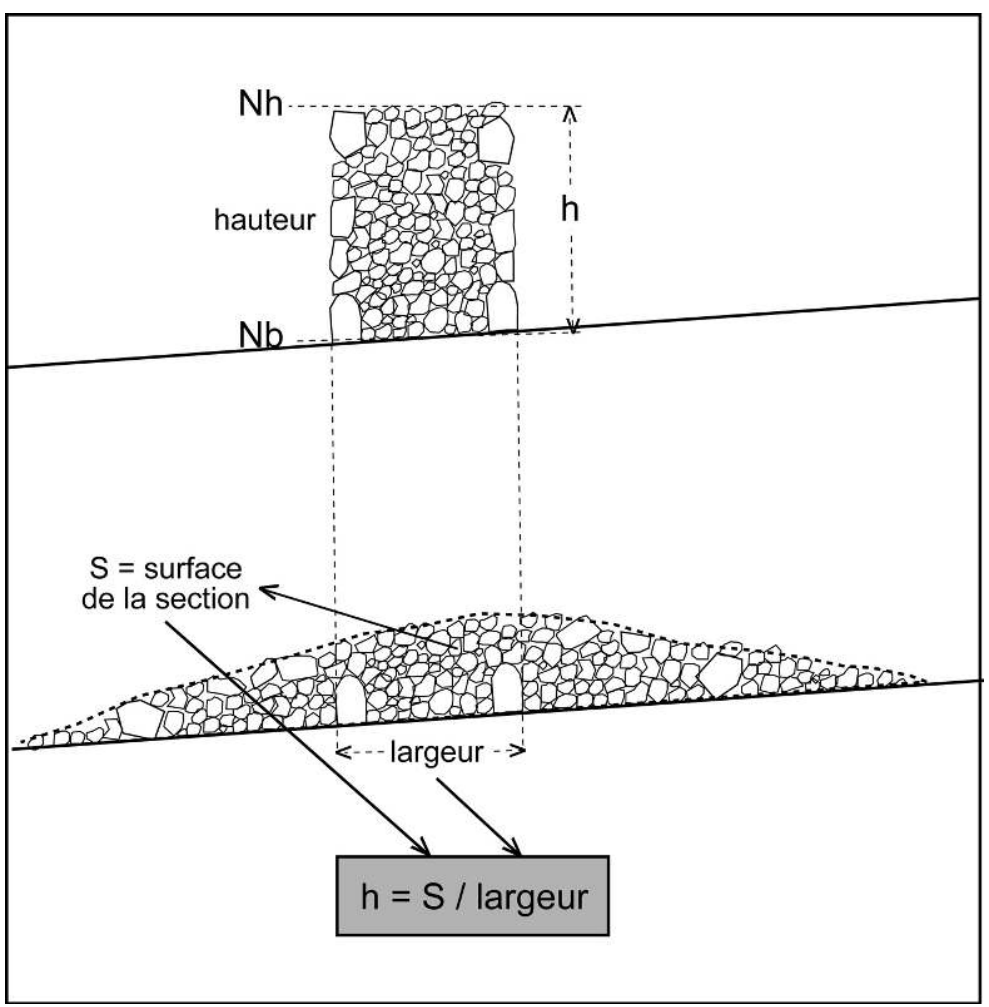

Nous avons vu supra qu'une double inégalité définit les possibilités d'implantation d'un barrage de pêcherie en vue d'une collecte maximale $\left(0,416_{*} M M \leq N_{b} \leq 0,583_{*} M M-h\right)$; bien entendu, pour les barrages établis en des temps anciens, ces contraintes nous renvoient à la période d'aménagement de ces barrages. En admettant que le marnage n'a pas changé entre-temps, l'utilisation du niveau de la base $\mathrm{N}_{\mathrm{b}}$ permet d'approcher les niveaux HMME et BMME anciens. En les comparant aux niveaux actuels, $\mathrm{HMME}_{\mathrm{a}}$ et $\mathrm{BMME}_{\mathrm{a}}$ on peut déterminer leurs variations à partir de $\delta$ (HMME) et de $\delta(\mathrm{BMME})$

$$
\begin{aligned}
& \delta(\mathrm{HMME})=\mathrm{HMME}_{\mathrm{a}}-\mathrm{N}_{\mathrm{b}}-\mathrm{h} \\
& \delta(\mathrm{BMME})=\mathrm{BMME}_{\mathrm{a}}-\mathrm{N}_{\mathrm{b}}
\end{aligned}
$$

\footnotetext{
marin entre l'époque d'édification du barrage concerné et l'actuel, qui peut correspondre aussi bien à la variation du niveau moyen $\mathrm{N}_{\mathrm{m}}$, soit $\mathrm{MM} / 2$, qu'à celui du niveau HMVE, puisque nous supposons une constance du marnage maximal dans le temps. Cette fourchette de valeurs de la variation du niveau marin n'est, en soi, pas suffisante, si on ne peut la rattacher à la chronologie de l'aménagement de la structure. C'est alors que le problème se complique car, ainsi qu'il a été évoqué plus haut, la construction de barrages en pierre demeure difficile à dater précisément, en l'absence de textes (nous ramenant, au mieux, à l'époque médiévale) et surtout sans des travaux de terrains plus approfondis ${ }^{5}$.
} 
archéch

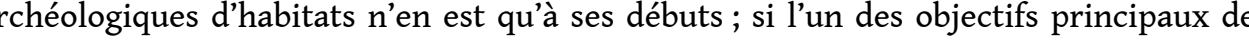
cette approche est l'identification des contextes chrono-culturels de ces barrages de pêcheries, elle constitue également une piste prometteuse pour déboucher sur une reconstitution de la variation du trait de côte et des niveaux marins durant l'Holocène, le long des côtes de la Bretagne. En l'état actuel de la recherche, tout laisse supposer que, parmi les centaines de pêcheries recensées sur le littoral breton, doivent exister des barrages remontant aux différentes périodes documentées par les nombreux sites archéologiques côtiers de la région. Nous allons analyser la situation pour le Bas-Léon et tester sur ce secteur géographique les possibilités et limites d'une approche chronologique théorique.

\section{Vers une approche théorique de la chronologie : un test sur les pêcheries du Bas-Léon}

Afin d'avancer dans la contextualisation chrono-culturelle des barrages de pêcheries, on peut évoquer la possibilité de dater les pêcheries lorsque, par ailleurs, on dispose de données fiables sur la variation du niveau marin dans une zone donnée. Cette approche a été menée de manière critique dans une zone-test (le littoral du Bas-Léon, Finistère, France) où, d'une part, de nombreux barrages ont été localisés et caractérisés (Daire, 
2008) et où, d'autre part, on dispose de mesures (positionnement altitudinal et dates radiocarbone) sur des dépôts littoraux (M.-Th. Morzadec-Kerfourn, 1969 ; 1974).

\section{Les barrages à poissons du Bas-Léon}

En excluant la dizaine de pêcheries de l'archipel de Molène et de Ouessant, 72 barrages à poissons en pierres subsistent sur le littoral de dix-sept communes du Bas-Léon; ce sont de véritables vestiges archéologiques connus actuellement avec assez de précision, (tableau 1) (Daire, 2008 ; Daire et Langouët, 2010, p. 83-121). Ils sont présentés ici, dans une progression géographique allant $\mathrm{du}$ sud (Plougonvelin) vers le nord-est (Plounéour-Trez), avec quelques-unes de leurs caractéristiques, extraites de la base de données PEB : les coordonnées Lambert II étendu, le niveau de la partie la plus basse des barrages $\left(\mathrm{N}_{\mathrm{b}}\right)$ mesuré par rapport au 0 SHOM (Service Hydrographique et Océanographique de la Marine), les traces toponymiques associées, la longueur des empierrements et la typologie (tableau 1). La cartographie des anciens barrages à poissons du Bas-Léon montre une concentration relative de ces installations entre l'Aber Ildut et Plouguerneau (fig. 4). Par contre, le littoral compris entre Plouarzel et la pointe Saint-Mathieu, d'une part et entre Plouguerneau et Brignogan, d'autre part, ne semble pas avoir été propice à l'implantation de pêcheries. Les barrages qui ont laissé un souvenir dans la toponymie littorale et marine (Pondaven, 1992) mais qui n'ont pas pu être retrouvés ou examinés, ne figurent pas dans cette liste. Au passage, on peut noter que le toponyme breton goret et ses variantes sont restés attachés aux vestiges de $26 \%$ des pêcheries identifiées.

Figure 4 : Répartition des 72 anciens barrages à poissons, connus à ce jour, sur le littoral du BasLéon (Finistère)/Distribution of the 72 considered fish-traps registered along the coasts of Bas-Léon (Finistère)
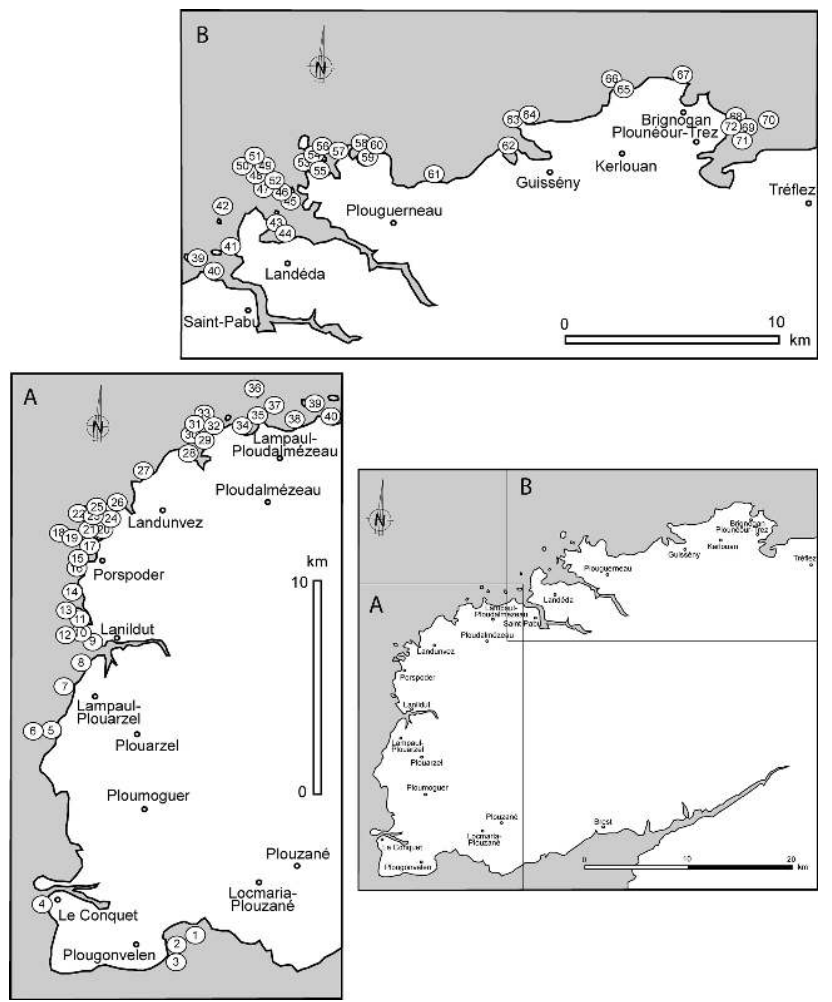
Les longueurs des barrages varient de 24 à $260 \mathrm{~m}$, en mettant à part trois barrages très longs ( $320 \mathrm{~m}$ pour les $\mathrm{N}^{\circ} 41$ et 70 et $348 \mathrm{~m}$ pour le $\mathrm{n}^{\circ} 31$ ) (tableau 1 , fig. 5 ). $63 \%$ des barrages ont une longueur comprise entre 40 et $140 \mathrm{~m}$ et $33 \%$ ont une longueur située entre 140 et $260 \mathrm{~m}$. À côté de ces grands barrages, dont la construction a dû nécessiter une main-d'œuvre importante et pour lesquelles on suppose une mobilisation communautaire, il existe quelques installations plus modestes, mais seuls $4 \%$ des pièges à poissons ont une longueur inférieure à $40 \mathrm{~m}$.

Figure 5 : Distribution des longueurs de 72 pièges à poissons du Bas-Léon (Finistère)/Distribution of the lengths of the fish-traps of Bas-Léon (Finistère)

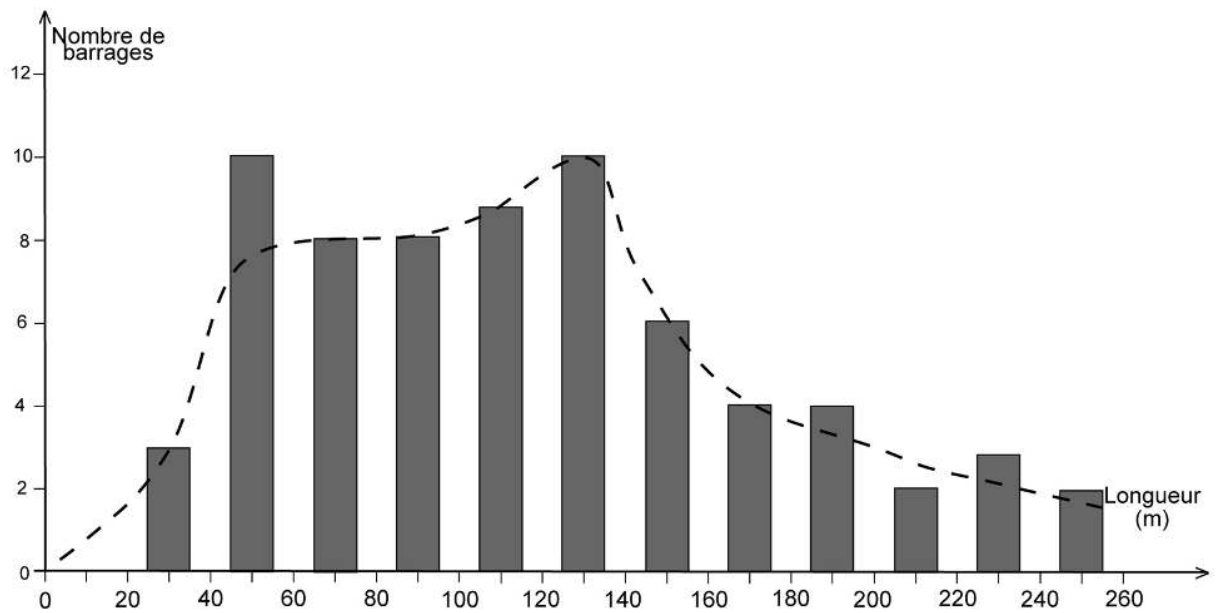
distingués en Bretagne d'après le plan des barrages et leur insertion dans l'estran (Daire et Langouët, 2008 ; Langouët et Daire, 2009). Le type A correspond à des barrages s'appuyant sur deux têtes ou massifs rocheux (photo 1); le type B combine plusieurs barrages de type $\mathrm{A}$, débouchant sur des installations possédant généralement un grand biez de pêche (photo 2).

Le type $\mathrm{C}$ est caractérisé par des plans en « $\mathrm{V}$ » où deux pannes ou bras se rejoignent en un point où était souvent fixée une nasse (ou un filet) (photo 3); le type D correspond à des pêcheries où l'une des pannes du type précédent est remplacée par la rive ellemême (photo 4); il en résulte que le pertuis est proche du rivage et facilement accessible. Trois sous-types de barrage ont été définis sur des critères géométriques par l'analyse du tracé des bras ou pannes : linéaire (L), courbe (C) ou sinueux (S). Ainsi, une pêcherie en « $\mathrm{V}$ » aux bras rectilignes sera classée dans le type $\mathrm{CL}$; un barrage constitué d'un empierrement incurvé s'appuyant sur deux roches sera dit du type AC.

Dans le Bas-Léon, le type A est nettement majoritaire ( $67 \%$ de ces pièges sont de ce type), que le barrage soit linéaire, incurvé ou éventuellement sinueux (1 cas). Aucun acte médiéval ou charte ne fournit de renseignement sur l'une de ces installations de pêche. Seule une mention de F. Le Masson du Parc concerne une pêcherie maritime de l'amirauté de Saint-Pol-de-Léon ou de Brest: «il y a au Corréjou de Trémeneach (Plouguerneau) un goret ou parc de pierres placé à l'entrée du port lequel on dit appartenir au Sieur Marquis de Kerouars, cette pêcherie est en décadence » (Levasseur, 2008), il s'agit des barrages à poissons $n^{\circ} 59$ et 60 qui forment un ensemble (tableau 1).

Norois, 220 | 2011 
F. Le Masson du Parc ne semble pas contester la légitimité de leur existence, ce qui signifierait que cette installation est antérieure à $1544^{6}$.

Photo 1 : Exemples de barrages à poissons de type A du Bas-Léon (@ Ortho littorale 2000)/ Examples of type $A$ fish traps in the Bas-Léon area (๑ Ortho littorale 2000)
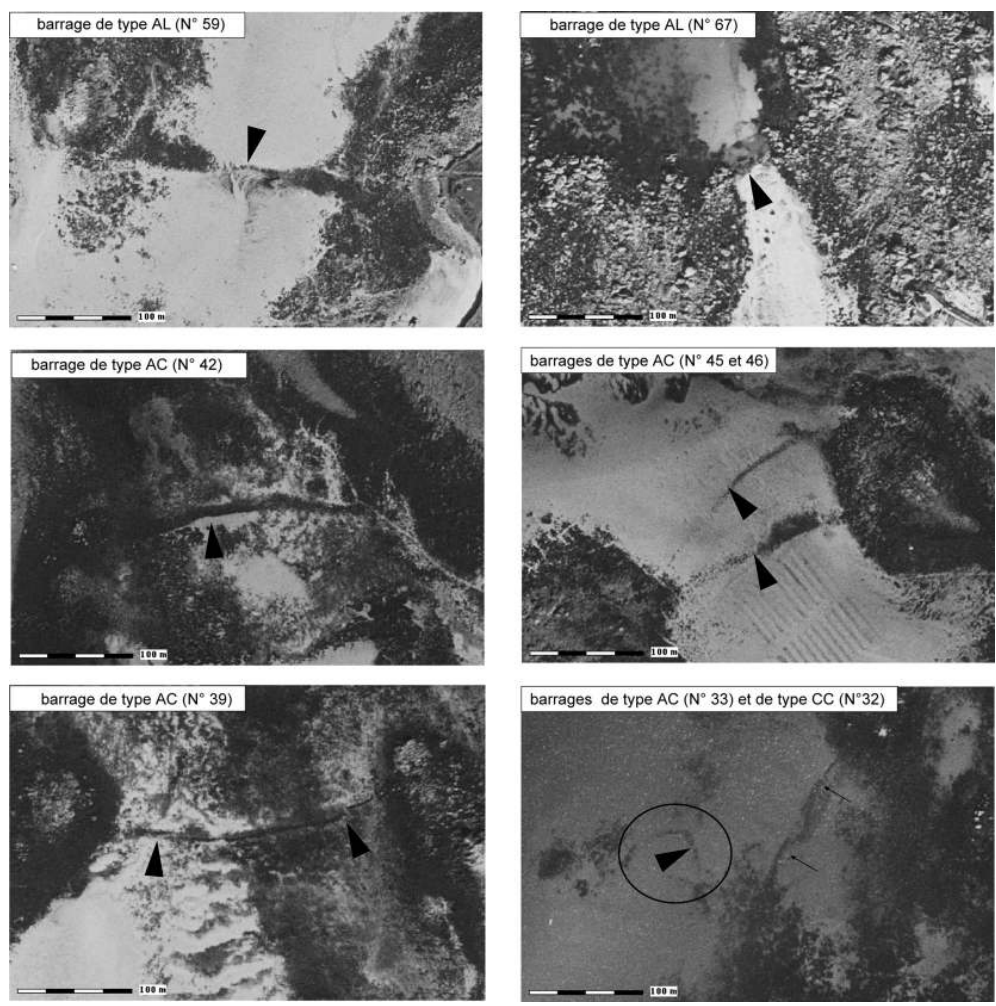

\section{Données sur la variation du niveau marin dans le Bas-Léon}

D’une manière générale, les travaux de M.-Th. Morzadec-Kerfourn (1969-1974), portant sur l'ensemble de la Bretagne, servent toujours de références dans les discussions sur les variations relatives du niveau marin au cours des derniers millénaires. En 1969 et 1974, ce chercheur avait étudié une série de tourbières réparties sur les côtes septentrionales du Finistère, du Conquet à Roscoff.

L'analyse de ces travaux sur la variation du niveau marin dans le nord Finistère débouche sur deux nécessités : celle de recourir à une calibration actualisée des dates radiocarbone afin de déboucher sur des dates calendaires et celle de revoir les calculs des niveaux marins à la lumière des données récentes du SHOM. En partant des mesures de comptage réalisées par G. Delibrias (Laboratoire de Gif/Yvette), le recours au logiciel en libre accès, OxCal (Laboratoire d'Oxford, Grande-Bretagne), débouche sur des intervalles de temps réels avec des coefficients de confiance à $95 \%$ (tableau 2, colonne «Datation Oxcal »). Pour certains sites, il n'y a pas de recouvrement entre les dates en «av. J.-C.» ou av. J.-C. (tableau 2, colonne « Date BP»), annoncées par M.Th. Morzadec-Kerfourn $(1969,1974)$ et les datations résultant de la calibration OxCal (tableau 2, zones grisées). En 1969 et 1974, la calibration avait consisté à diminuer simplement la date BP de 1950 ans, 1950 étant l'année définie comme référence lors de la Conférence de Cambridge (1962). L'explication en est simple: les premières calibrations ont été vulgarisées par la publication de tables en 1982 (Klein et al., 1982), 
soit plusieurs années après les publications de M.-Th. Morzadec-Kerfourn. De toute manière, les intervalles de mesure donnés par le Laboratoire de Gif/Yvette correspondaient à un écart-type (soit un coefficient de confiance à $66 \%$ ). Actuellement on utilise deux écarts-types pour obtenir une datation avec un coefficient de confiance de $95 \%$.

Photo 2 : Exemple d'une pêcherie de type $B$, avec trois barrages (Plounéour-Trez, Goueltoc, $\mathrm{n}^{\circ} 70$ ) (๑ Ortho littorale 2000)/Example of type B fish trap, with three dams (Plounéour-Trez, Goueltoc, $n^{\circ} 70$ ) (๑) Ortho littorale 2000)

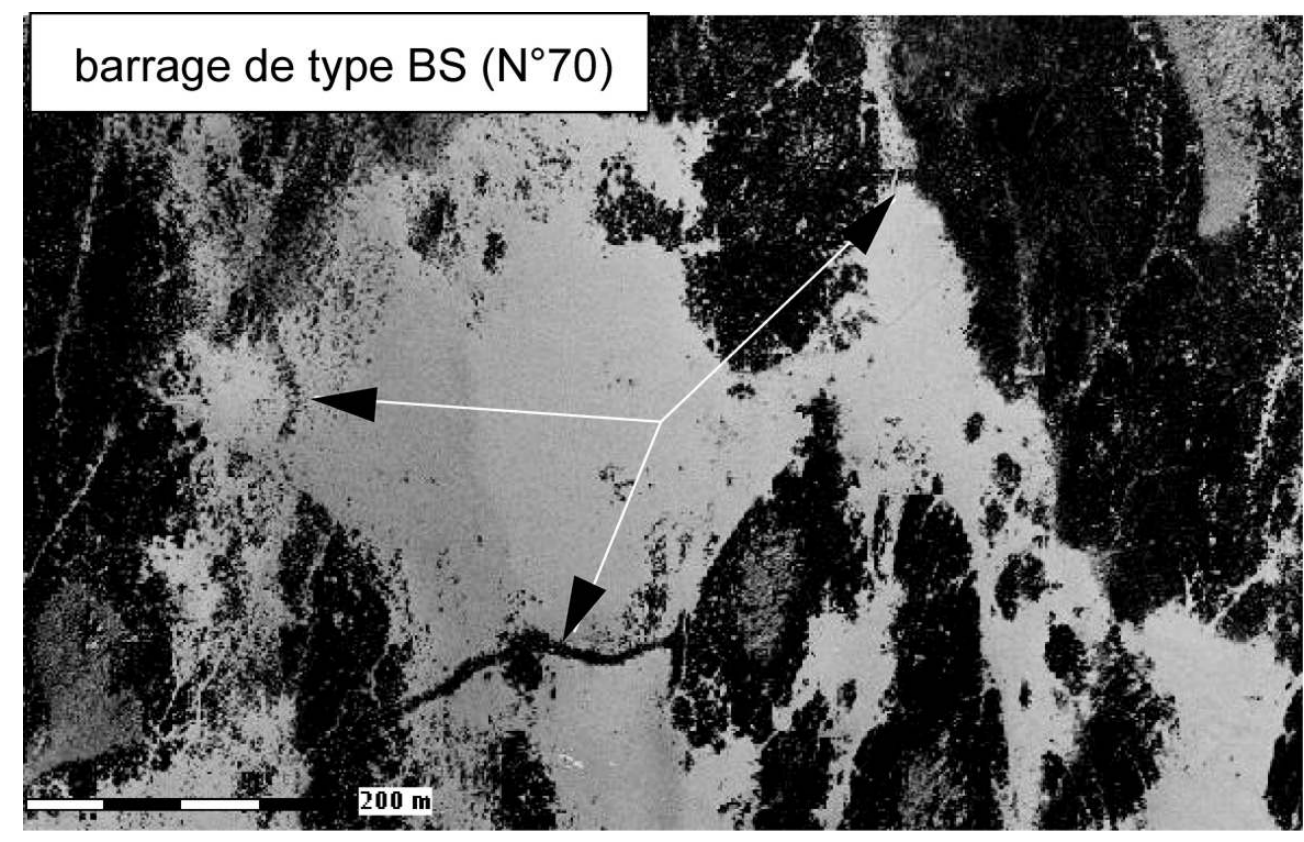

Photo 3 : Exemples de barrages à poissons de type $C$ du Bas-Léon (๑ Ortho littorale 2000)/ Examples of type $C$ fish traps in the Bas-Léon area (๑ Ortho littorale 2000)
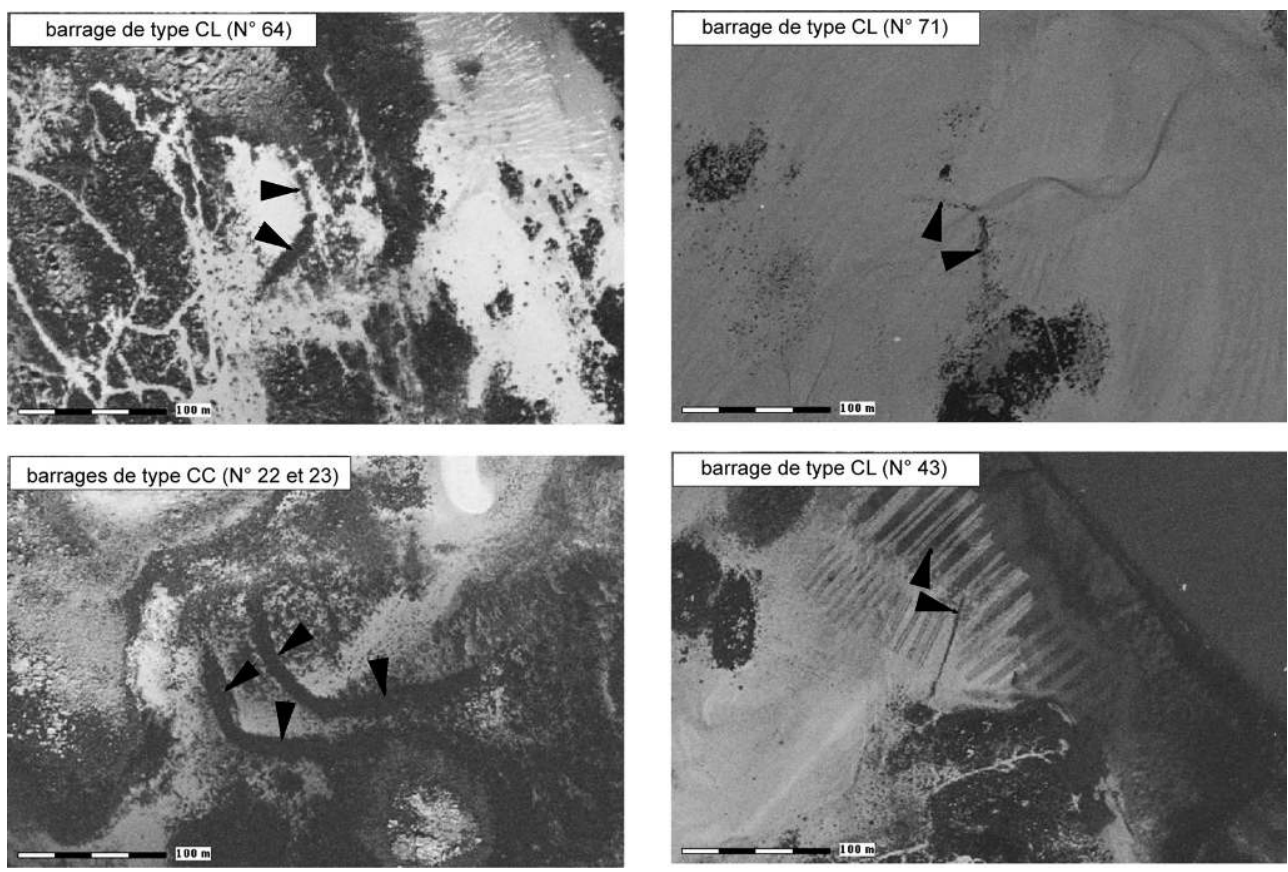
Photo 4 : Exemples de barrages à poissons de type $D$ du Bas-Léon (๑ Ortho littorale 2000)/ Examples of type $D$ fish traps in the Bas-Léon area (๑ Ortho littorale 2000)
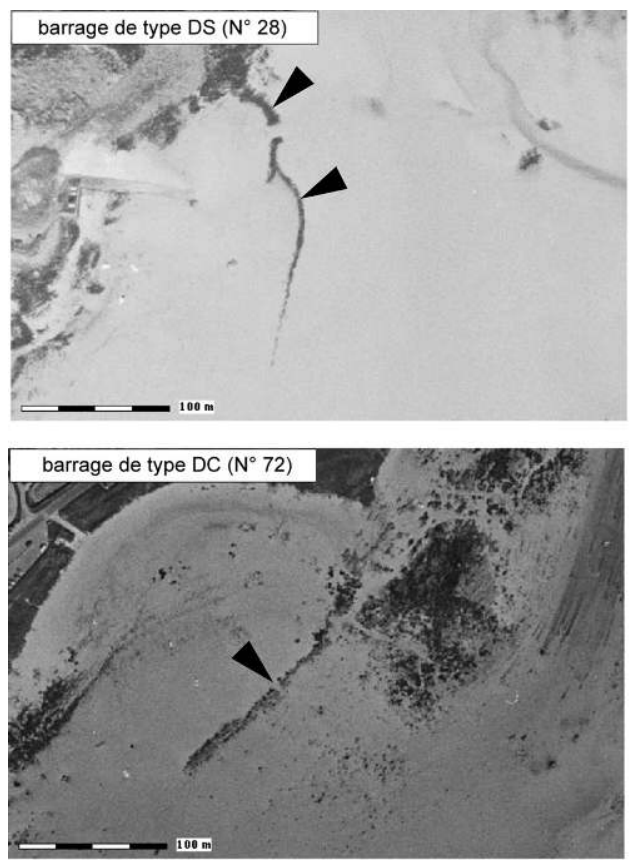
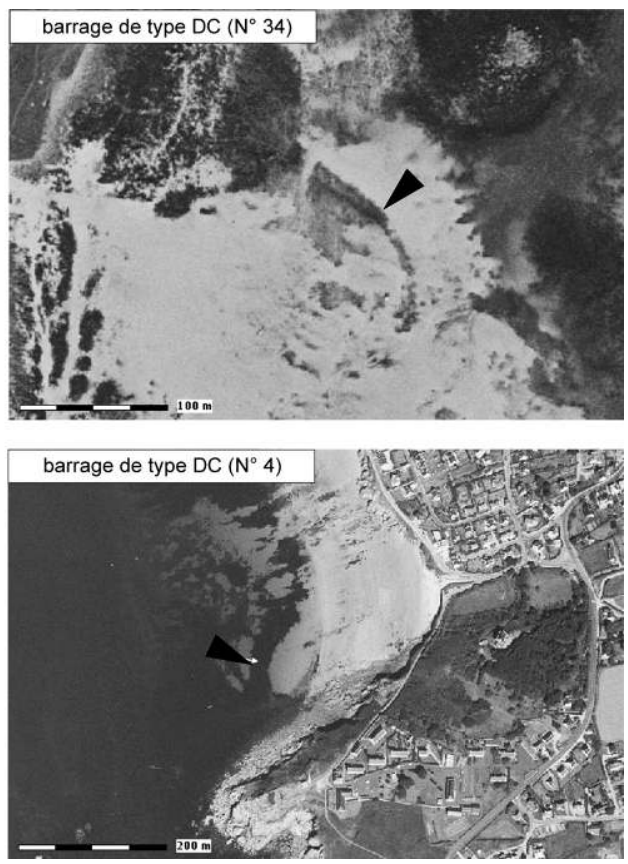

Dans les graphiques illustrant les variations relatives du niveau de la mer (MorzadecKerfourn, 1969, 1974), les datations radiocarbone figurées par des points auraient du être représentées avec une barre d'imprécision, même sur une échelle en temps BP. A. Pirazzoli attribue en partie à cette anomalie le caractère oscillatoire des courbes de M.T. Morzadec-Kerfourn : " in the absence of clearly establihed error boxes for each sea-level index point used, apparent oscillations may simply result from underestimed locally larger uncertainty ranges" (Pirazzoli, 1991). Par ailleurs, les niveaux des prélèvements effectués par M.-Th. Morzadec-Kerfourn, présentés par rapport au 0 NGF $(1969,1974)$ restent précieux. En revanche, leurs transformations par rapport au 0 SHOM ou par rapport au niveau des plus hautes mers de vive-eau (HMVE) doivent être révisées à la lumière des tables SHOM actuellement disponibles. En effet pour une cinquantaine de sites côtiers bretons, on dispose aujourd'hui de la différence locale entre les 0 SHOM et IGN, d'une part, et des marnages maximaux locaux, c'est-à-dire des niveaux HMVE, d'autre part. Après calculs, on a pu constater des différences de repérage, par rapport au niveau des plus hautes mers de vive-eau, entre les nouvelles valeurs (tableau 3, colonne "niveau HMVE-LL ») et celles de M.-Th. Morzadec-Kerfourn (tableau 3, colonne "niveau HMVE-MTMK »); ces dernières sont systématiquement supérieures aux valeurs réelles, ce qui est très gênant en regard d'un objectif de recherche visant à évaluer la variation du niveau marin durant les derniers millénaires. Les nouvelles valeurs, proposées ici, qui résultent de progrès faits dans le repérage local du 0 SHOM par rapport au 0 NGF, ne peuvent qu'être prises en compte de nos jours.

Tableau 2 : Révision des datations des prélèvements effectués dans des tourbières du nord Finistère/Revision of the North Finistère peat bog samplings dating

\begin{tabular}{|l|l|l|l|l|l|}
\hline $\mathrm{N}^{\circ}$ & Site & Réf. datation & Date BP & Date MTMK & Datation Oxcal \\
\hline 1 & Argenton (A) & GIF 817 & $2180 \pm 105$ & $230 \mathrm{BC}$ & $482 \mathrm{BC}-60 \mathrm{AD}$ \\
\hline
\end{tabular}




\begin{tabular}{|l|l|l|l|l|l|}
\hline 2 & Santec & GIF 818 & $2330 \pm 105$ & $380 \mathrm{BC}$ & $758-111 \mathrm{BC}$ \\
\hline 3 & Lampaul-Plouarzel & GIF 712 & $3020 \pm 110$ & $1070 \mathrm{BC}$ & $1500-941 \mathrm{BC}$ \\
\hline 4 & Plouescat (S) & GIF 710 & $3390 \pm 120$ & $1440 \mathrm{BC}$ & $2012-1432 \mathrm{BC}$ \\
\hline 5 & Argenton (B) & GIF 815 & $3620 \pm 125$ & $1665 \mathrm{BC}$ & $2400-1665 \mathrm{BC}$ \\
\hline 6 & Trézien & GIF 714 & $3660 \pm 115$ & $1710 \mathrm{BC}$ & $2454-1742 \mathrm{BC}$ \\
\hline 7 & Argenton (C) & GIF 816 & $3970 \pm 35$ & $2020 \mathrm{BC}$ & $2577-2348 \mathrm{BC}$ \\
\hline 8 & Plouescat (B) & GIF 711 & $4120 \pm 140$ & $2170 \mathrm{BC}$ & $3086-2286 \mathrm{BC}$ \\
\hline 9 & Plouguerneau & GIF 282 & $4250 \pm 250$ & $2285 \mathrm{BC}$ & $3626-2202 \mathrm{BC}$ \\
\hline 10 & Brignogan (Cha.Ble.) & GIF 75 & $4980 \pm 120$ & $3030 \mathrm{BC}$ & $4042-3521 \mathrm{BC}$ \\
\hline 11 & Ploudalmézeau & GIF 766 & $5770 \pm 150$ & $3820 \mathrm{BC}$ & $4988-4341 \mathrm{BC}$ \\
\hline
\end{tabular}

Comparer les datations radiocarbone BP (colonne 4) (Morzadec-Kerfourn, 1969 et 1974) et les dates nouvellement calibrées (colonne 6).

Compare radiocarbon BP dating (column 4) (Morzadec-Kerfourn, 1969 et 1974) and newly calibrated dates (column 6).

Tableau 3 : Révision des niveaux topographiques des prélèvements effectués dans des tourbières du nord Finistère (Morzadec-Kerfourn, 1969 et 1974)/Revision of the topographic levels for the North Finistère peat bog samplings (Morzadec-Kerfourn, 1969 et 1974)

\begin{tabular}{|l|l|l|l|l|l|l|}
\hline $\mathrm{N}^{\circ}$ & Site & $\begin{array}{l}\text { Datation } \\
\text { Oxcal }\end{array}$ & $\begin{array}{l}\text { niveau } \\
/ 0 \mathrm{NGF}\end{array}$ & $\begin{array}{l}\text { niveau } \\
/ 0 \text { SHOM }\end{array}$ & $\begin{array}{l}\text { niveau } \\
\text { /HMVE } \\
\text { (MTMK) }\end{array}$ & $\begin{array}{l}\text { niveau } \\
\text { /HMVE } \\
(\mathrm{LL})\end{array}$ \\
\hline 1 & Argenton-(A) & $482 \mathrm{BC}-60 \mathrm{AD}$ & $+4,5$ & 8,4 & $+0,6$ & 0 \\
\hline 2 & Santec & $758-111 \mathrm{BC}$ & $+2,0$ & 6,8 & $-2,8$ & $-3,0$ \\
\hline 3 & Lampaul-Plouarzel & $1500-941 \mathrm{BC}$ & $+3,5$ & 7,2 & 0 & $-0,8$ \\
\hline 4 & Plouescat (S) & $2012-1432 \mathrm{BC}$ & $+2,5$ & 6,9 & $-2,2$ & $-2,2$ \\
\hline 5 & Argenton-(B) & $2400-1665 \mathrm{BC}$ & 0 & 3,9 & $-3,9$ & $-4,5$ \\
\hline 6 & Trézien & $2454-1742 \mathrm{BC}$ & $+1,5$ & 5,0 & $-2,2$ & $-2,7$ \\
\hline 7 & Argenton (C) & $2577-2348 \mathrm{BC}$ & $-0,5$ & 3,4 & $-4,4$ & $-5,0$ \\
\hline 8 & Plouescat (B) & $3086-2286 \mathrm{BC}$ & $+2,0$ & 6,4 & $-2,7$ & $-2,7$ \\
\hline 9 & Plouguerneau & $3626-2202 \mathrm{BC}$ & $+4,0$ & 8,1 & $-0,5$ & $-0,4$ \\
\hline 10 & Brignogan (Cha. Ble.) & $4042-3521 \mathrm{BC}$ & $+3,0$ & 7,4 & $-1,7$ & $-1,7$ \\
\hline 11 & Ploudalmézeau & $4988-4341 \mathrm{BC}$ & $-4,4$ & $-0,2$ & $-8,8$ & $-9,0$ \\
\hline 12 & Kerbors & $204 \mathrm{BC}-74 \mathrm{AD}$ & $+4,4$ & 9,7 & - & $-1,3$ \\
\hline & Sites datés par interpolation des analyses et dates polliniques & \\
\hline 13 & Brignogan (Scluz) & $2577-1742 \mathrm{BC}$ & $+2,5$ & 6,9 & $-2,2$ & $-2,3$ \\
\hline 14 & Porspoder & $2012-941 \mathrm{BC}$ & $+4,2$ & 7,9 & $+0,3$ & $-0,3$ \\
\hline 15 & Goulven & $2202-3626 \mathrm{BC}$ & $+1,7$ & 6,1 & $-3,0$ & $-3,0$ \\
\hline
\end{tabular}




\begin{tabular}{|l|l|l|l|l|l|l|}
\hline 16 & Plounéour-Trez & $2400-1432 \mathrm{BC}$ & +2 & 6,4 & $-2,7$ & $-2,7$ \\
\hline
\end{tabular}

La colonne 3 donne les datations révisées (cf. tab. 2) et la colonne 7, les niveaux corrigés.

Column 3 gives the newly calibrated dating (cf. tab. 2) and column 7, the newly calculated levels.

Lorsque, pour certains prélèvements, il y avait conflit entre les datations radiocarbone et résultats des analyses polliniques, M.Th. Morzadec-Kerfourn a privilégié l'interpolation des analyses polliniques en suspectant une contamination des échantillons par des racines intrusives, comme ce fut le cas pour les échantillons de Porspoder et Goulven (tableau 3). Il nous a été possible de « récupérer » ces données en utilisant les datations radiocarbones, étendues et combinées, de sites encadrant chronologiquement les lieux de prélèvement à problème. Cela a permis de densifier le graphique, mais en fait n'apporte rien de neuf puisque cela revient à introduire des interpositions moyennées. Sur le graphique corrigé des dates calibrées et des niveaux de prélèvements, les sites de tourbière à eau saumâtre se distinguent par un rectangle blanc (fig. 6) ; ils sont plus significatifs dans l'établissement d'une courbe-enveloppe devant passer sous tous les points représentatifs. Les nouvelles valeurs des niveaux repérés par rapport à (HMVE) $)_{\text {actuel }}$ et les datations radiocarbone nouvellement calibrées donnent naissance à un graphique permettant de proposer une courbe de variation du niveau marin $\delta$ (HMVE), dessinée a minima (fig. 6), en fonction d'une échelle calendaire. Or, un niveau de HMVE est connu un peu plus à l'est (Kerbors, Côtes d'Armor) grâce à une observation directe (Langouët et Goulpeau, 1980): les vestiges d'un foyer de briquetages se trouvaient à $1,70 \mathrm{~m}$ sous le niveau (HMVE) actuel et la datation radiocarbone (GIF 2527) a donné le résultat $2050 \pm 60 \mathrm{BP}$, soit 204 av. J.-C. $-74 \mathrm{AD}$. Le résultat correspondant au site de Santec apparaît comme anormal (fig. 6) or son niveau de prélèvement semble correct ; ce serait plutôt la datation radiocarbone qui aurait pu être erronée, la tourbière d'origine ayant pu être perturbée par des matières organiques plus récentes. 
Figure 6 : Courbe hypothétique de variation du niveau HMVE dans le Bas-Léon pour les sept derniers millénaires/Hypothetic curve figuring the highest tides level variations (HMVE) in the Bas-Léon area for the last seven thousand years

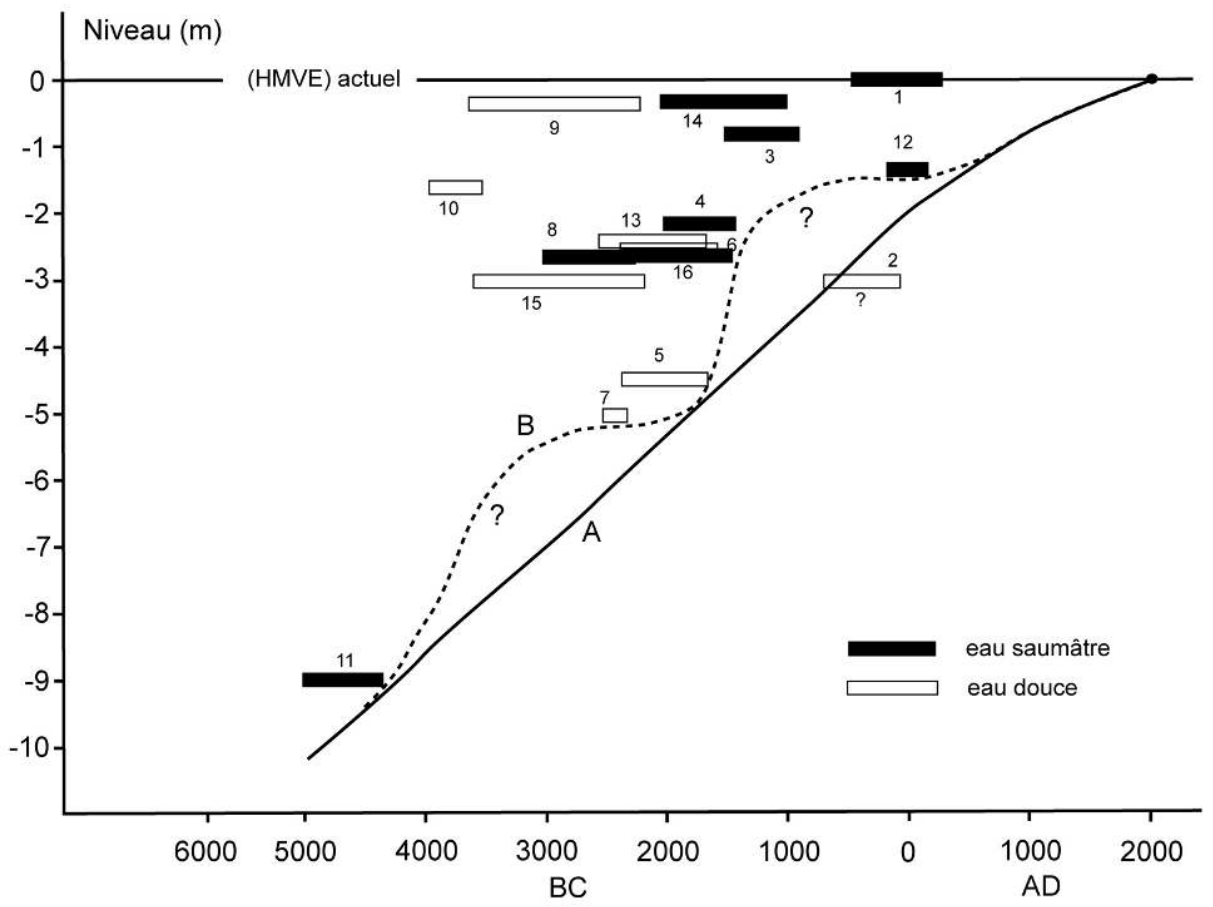

Ne serait-ce que par son caractère apériodique ou monotone, la courbe proposée A (en trait plein fig. 6) ne ressemble pas à celles de M.-Th. Morzadec-Kerfourn $(1969,1974)$. A. Pirazzoli s'étonnait du caractère oscillatoire des courbes obtenues pour la Bretagne et en attribuait l'origine aux échantillonnages utilisés. De fait, les courbes de I. Shennan (1989) obtenues pour le sud-ouest de la Grande-Bretagne sont plus régulières (Giot, 1990). Mais, à datations égales, les niveaux des prélèvements reportés par rapport au niveau HMVE actuel sont un peu plus bas (tableau 3). Par ailleurs, à niveau de prélèvement égal, les nouvelles datations radiocarbone calibrées sont très souvent plus anciennes que celles proposées dans la publication de 1969 (Morzadec-Kerfourn, 1969) (tableau 2). En résumé toutes les données de références se sont déplacées dans le nouveau graphe. La courbe proposée pour la variation du niveau marin HMVE ne présente plus la régression située après 1000 av. J.-C. On pourrait à l'évidence proposer une courbe légèrement sinueuse qui colle au mieux des points, mais cela compliquerait la lecture et ne permettrait pas de retrouver une régression très sensible autour de 1000 av. J.-C. (fig. 6, courbe tiretée). Les travaux récents de P. Stéphan (ce volume) ont débouché sur l'établissement d'une courbe des variations relatives des niveaux marins en Bretagne occidentale qui diffère sensiblement de celle, modifiée, de M.-Th. Morzadec-Kerfourn (fig. 6). D'une manière générale, la courbe de P. Stéphan montre des variations des niveaux marins d'amplitude moindre de 2 à $3 \mathrm{~m}$; par ailleurs un phénomène régressif important est mis en évidence autour de $1000 \mathrm{av}$. J.-C., phénomène qui a pratiquement disparu sur la courbe de M.-Th. Morzadec-Kerfourn après calibration des dates radiocarbone (fig. 6). En attendant la suite de ces travaux, nous nous sommes contentés de la courbe A de la figure 6. M.-Th. Morzadec-Kerfourn avait également utilisé comme points de références des monuments mégalithiques se 
trouvant sur les estrans, mais nous ne connaissons pas les datations utilisées pour les placer dans l'échelle du temps. Y a-t-il eu des datations radiocarbone? Est-ce que ce sont des indications de chronologie relative (du type « Néolithique moyen ») qui ont été indirectement utilisées pour un calage chronologique ? C'est difficile de le dire. De toute manière, ces jalons ne semblent pas avoir contribué d'une manière fondamentale au tracé de la courbe de 1969 (Morzadec-Kerfourn, 1969).

La courbe proposée aujourd'hui et tracée à minima (A, fig. 6), qui représente la différence de hauteur $\delta(\mathrm{HMVE})$ en fonction du temps peut se formuler ainsi :

$\delta(\mathrm{HMVE})=0,00176_{*} t+1,71$

où $\delta$ (HMVE) est exprimé en mètre et $t$, en année av. J.-C.. Par exemple, en 2000 av. J.-C., le niveau ancien de la HMVE, d'après cette formulation, aurait été de $-5,23 \mathrm{~m}$ par rapport au niveau actuel.

51 En utilisant cette courbe monotone et linéaire et en la complétant par extrapolation jusqu'au présent, on peut proposer une courbe simplifiée de variation du niveau HMVE dans le Bas-Léon pour les six derniers millénaires (courbe A, fig. 6).

\section{Confrontation des données}

52 La prise en compte des niveaux d'implantations des barrages à poissons du Bas-Léon, l'utilisation des règles d'accessibilité préalablement établies, et le recours à la variation du niveau marin valable pour la même zone géographique (fig.6), permettent de proposer, pour chaque pêcherie, une fourchette chronologique de son aménagement initial (fig. 7). La datation est donnée avec des précisions allant de \pm 500 ans à \pm 700 ans.

Les premiers barrages semblent apparaitre au cours du III millénaire av. J.-C.; il est cependant probable que certains pièges à poissons très anciens ( $\mathrm{du}$ Mésolithique et du début du Néolithique par exemple) restent indécelables à ce jour sur les vues aériennes car localisés à des profondeurs trop grandes : en effet, même lorsque les vues aériennes ont été prises à très basse marée, la limite de visibilité est de l'ordre de $2 \mathrm{~m}$ sous le 0 SHOM. En distinguant trois grandes périodes et en ne prenant en compte que les pièges dont la chronologie est clairement incluse, sans ambiguïté, dans l'une de ces trois périodes, des cartographies "chronologiques" sont proposées et suscitent quelques commentaires (fig. 8). Ces cartographies recouvrent respectivement les périodes 3000-2 000 av. J.-C., 2000 av. J.-C.-0, 0-2 000 AD. A-t-il existé, dans cette zone côtière, des pêcheries plus anciennes? Si oui, pourquoi n'ont elles pas été détectées ? D'après la figure 6, le niveau HMVE aurait monté d'environ $11 \mathrm{~m}$ depuis la fin du Mésolithique. Or, pour le Bas-Léon, les marnages maximaux passent de 7,8 $\mathrm{m}$ au Conquet à $10,5 \mathrm{~m}$ à Roscoff, ce qui signifie que les potentiels barrages "mésolithiques" devraient se trouver approximativement à des niveaux compris entre $6,6 \mathrm{~m}\left(11-0,416^{*} 10,5\right)$ et $7,7 \mathrm{~m}$ $\left(11-0,416^{*} 7,8\right)$ sous le zéro des cartes marines. On comprend bien que ces vieux barrages ne puissent pas être détectés d'avion ou de satellite et que l'échantillonnage des pièges à poissons du Bas-Léon, réuni dans cette étude grâce à une exploitation de photographies aériennes, a une limite chronologique inférieure. Pour la période allant de 3000 à 2000 av. J.-C., les pièges à poissons du Bas Léon sont implantés principalement dans le secteur des abers (globalement entre l'aber Ildut et l'aber Benoit), et plus sur les côtes nord-ouest du Bas-Léon. Mais, cette période, imposée par les méthodes de recherches, ne recouvre pas la Préhistoire, mais seulement la fin du Néolithique. Il est donc délicat de comparer la répartition de pêcheries datées d'entre 
3000 et 2000 av. J.-C. avec celle de la période suivante (de 2000 av. J.-C. à 0). Pour cette dernière période, la distribution montre deux concentrations de pièges: l'une, relativement dense, entre l'Aber Ildut et Landunvez, l'autre autour des embouchures de l'Aber Benoit et de l'Aber Vrac'h. Pour la période historique (de 0 à $2000 \mathrm{AD}$ ), les aménagements de pièges s'égrènent sur toute la côte du Bas-Léon sans concentration particulière.

Figure 7 : Datations des barrages à poissons du Bas-Léon à partir des mesures de leurs niveaux d'implantation dans l'estran. Les rectangles sombres correspondent à des groupes de plusieurs pêcheries présentant la même datation/Bas-Léon fish traps dating from the measure of their weir layout. The dark rectangles correspond to groups of several fish traps sharing the same dating

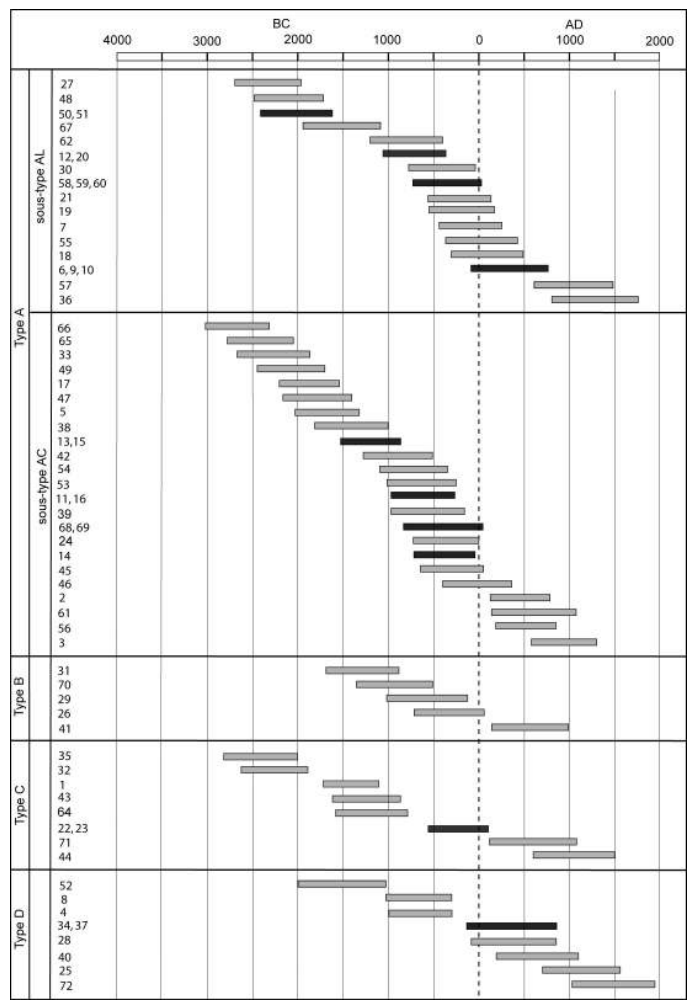

La distribution des dates en fonction du type de barrage indique qu'il n'y a pas eu d'évolution typologique (fig. 7) : de 3000 av. J.-C. (Néolithique récent) au Moyen Âge, tous les types de barrages sont figurés. Toutefois, on peut noter que les structures de type B, constituées en fait de plusieurs barrages de type A, apparaissent plus tardivement que les types A ou C ; leur mise en place nécessitait une main-d'œuvre plus importante et, à l'évidence, une certaine organisation sociale. Les traces toponymiques sont associées à des pièges à poissons qui, quel que soit leur type, ne remontent pas à des périodes antérieures au début du $I^{\text {er }}$ millénaire av. J.-C. (fig. 9). Ces quelques observations d'ordre chrono-culturelles sont-elles cohérentes avec les données archéologiques sur l'occupation humaine du Bas-Léon?

Les données archéologiques révèlent une constance de l'occupation humaine sur le territoire qui est aujourd'hui le bas plateau du Léon (Giot et al., 1998; Tanguy et Lagrée, 2002). Pour le Pléistocène, ces vestiges d'implantations anthropiques ne peuvent être reliés au milieu maritime ni à des activités côtières, compte tenu d'une géographie très différente en lien avec les variations de niveaux marins. Mais ces indices anthropiques 
indiquent néanmoins que le secteur géographique était occupé et rien n'interdit de penser qu'ils se prolongeaient dans les secteurs aujourd'hui immergés.

Les grands monuments mégalithiques du Léon, et en particulier les complexes de Barnenez, Carn et Guennoc ont connu leurs premières phases d'édification aux $5^{\mathrm{e}}-4^{\mathrm{e}}$ millénaires av. J.-C.; préservés sur des points relativement hauts, ils constituent les traces de communautés humaines et d'activités qui se sont développées sur la plaine littorale. Même si elles ne nous sont pas accessibles en l'état actuel des recherches, il est probable que des installations de pêcheries ont pu apporter un complément alimentaire à ces communautés d'agriculteurs et de bâtisseurs de monuments mégalithiques : cette capacité à mobiliser une main-d'œuvre conséquente autour de projets architecturaux communautaires est précisément requise dans le cas de pêcheries d'envergure, ainsi que le montrent les données d'époques historiques (voir notamment le cas des communautés religieuses). Il pourra être intéressant d'analyser, pour d'autres espaces géographiques, cette corrélation entre le développement du mégalithisme et celui des installations de pêcheries.

Les grands monuments évoqués, ont été agrandis, remaniés et utilisés pendant de longues périodes: le sanctuaire mégalithique de Barnenez a été en usage jusque vers 2000 av. J.-C.. La sépulture de l'île Carn était encore utilisée vers 2500 av. J.-C. Quant au site de l'île Guennoc, le monument mégalithique fut utilisé, dans sa fonction funéraire pendant 3 millénaires mais le site fut réoccupé au cours $d u \mathrm{II}^{\mathrm{e}}$ et surtout $\mathrm{I}^{\mathrm{er}}$ millénaire av. J.-C. en tant qu'habitat, lieu de vie et de travail. Ces pêcheries, si densément reconnues dans le secteur des abers pour la période $3000-2000 \mathrm{av}$. J.-C. et plus encore pour les deux derniers millénaires av. J.-C. (fig. 8), peuvent donc être liées aux différentes formes d'occupations reconnues, qui touchent principalement le domaine funéraire pour les phases les plus anciennes (sépultures mégalithiques puis série des grands tumulus du Léon). Il est probable que la présence de ressources métallurgiques, en particulier stannifères (à Saint-Renan, Bourg-Blanc) a entraîné le développement de nouveaux foyers de peuplement ou le renforcement de structures existantes dans ce secteur du Léon, au cours des Âges du Bronze et du Fer. Une récente étude a montré la densité du peuplement de ce secteur géographique du Bas Léon au cours du $1^{\mathrm{er}}$ millénaire av. J.-C., avec 350 sites ou gisements archéologiques de natures variées (habitats, sites artisanaux, sépultures, etc.) (Daire et al., 2011). 


$$
E
$$


59 Alors que les phases anciennes font apparaître des concentrations des pêcheries, pour la période postérieure au changement d'ère, la figure 8 montre une distribution spatiale beaucoup régulière des vestiges de barrages le long de la côte. Globalement, on pourrait voir dans ce changement une répercussion de la structuration du territoire en paroisses, puis en communes, avec un contrôle religieux ou civil centralisé des exploitations. À titre d'hypothèse, nous proposons donc d'envisager que l'implantation de pêcheries côtières, à différentes périodes préhistoriques, protohistoriques et historiques, puissent refléter les modalités de peuplement, voire servir d'indicateur sur la structure sociale des communautés, en tant que projet architectural mobilisant une main-d'œuvre relativement abondante (au même titre que la construction d'un monument mégalithique ou d'une église paroissiale) et en tant que source de revenu pouvant bénéficier à un groupe humain plus ou moins important.

\section{Conclusions et perspectives}

Nous avons souligné les principes de corrélation entre les implantations des barrages à poissons et les niveaux marins, les premières ayant fatalement évolué au rythme des secondes. Cette corrélation est à même d'expliquer les différences des niveaux de ces pêcheries telles que nous les retrouvons aujourd'hui, différences traduisant l'évolution chronologique de cette forme d'exploitation des ressources marines côtières. Les mesures et les datations faites sur des sites côtiers du Bas Léon par M.-Th. MorzadecKerfourn, même s'il a fallu les actualiser, ont permis d'obtenir des datations des anciens pièges à poissons de cette même zone, grâce à des réflexions fondamentales sur les règles d'aménagement.

61 La démonstration et la validation du schéma ont été possibles dans le cas du Bas-Léon, mais toutes les zones littorales ne sont pas actuellement aussi bien documentées, en particulier en matière de jalons chronologiques et topographiques sur les variations des niveaux marins holocènes. Par son approche à la fois théorique et méthodologique, cette étude marque donc la première avancée dans un processus interdisciplinaire qui s'inscrit dans le moyen, voire le long terme et dont les résultats intéressent autant les archéologues que les environnementalistes des zones côtières.

Nous exprimons notre réelle gratitude aux deux relecteurs anonymes du présent article qui nous ont permis, grâce à leur travail consciencieux, précis et positif, d'améliorer notre texte et certaines illustrations et de corriger certaines fautes ou passages. Nous adressons également un grand remerciement au Laboratoire Costel (UMR 6554) qui a mis à notre disposition toutes ses collections de couvertures aériennes (IGN, IFREMER, Équipement, etc.). 


\section{BIBLIOGRAPHIE}

BELL M., 2007. Investigating and managing the coastal archaeological resource for a sustainable future. Lessons from the Severn estuary, Wales and England. Abstract book of the $13^{\text {th }}$ International Congress of the EAA (European Association of Archaeologists), Zadar (Croatia), p. 204-205.

BeLL M., CASELDINE A. NEUMAHN, H., 2000. Prehistoric intertidal archaeology in the Welsh Severn Estuary, York, CBA Research Report, 120.

BILLARD C., BERNARD V., BOUFFIGNY A., QUÉVILLON S., 2006. Archéologie des pêcheries dans la partie nordorientale de la baie du Mont-Saint-Michel. Journée Civilisations atlantiques et Archéosciences, Rennes, 8 avril 2008, UMR C2A, Université de Rennes 1, p. 61-63.

COLLECTIF, 1983. Les tableaux de correction des dates C 14, effectués par le Groupe de Tucson, Revue d'Archéométrie, Supplément C 14, 99 p.

COOPER J., 2005. Fish weir structures found in Southampton water, Nautical Archaeology Society Newsletter, $\mathrm{n}^{\circ} 3-4$, p. 4-8.

DAIRE M.-Y., 2001. Un hameau armoricain de la fin de l'Age du Fer sur l'île d'Yoc'h. dans ColLis J. (dir.), Society and settlement in Iron Age Europe, Actes du XVIII ${ }^{\mathrm{e}}$ Colloque de l'AFEAF, Winchester, april 1994. Ed. J. Collis, Université de Sheffield, p. 159-189.

DAIRE M.-Y., 2008. Des pêcheries d'estran sur les côtes du Bas Léon (Finistère), dans DAIRE M.-Y. et LANGOÜ̈t L. (dir.), Les pêcheries de Bretagne, Archéologie et Histoire des pêcheries d'estran, Coéd. CeRAA/AMARAI, Les Dossiers du Centre Régional d'Archéologie d'Alet, n AE, p. 113-121.

DAIRE M.-Y., LANGOUËT L. (dir.), 2008a. Les pêcheries de Bretagne, Archéologie et Histoire des pêcheries d'estran, Coédition CeRAA/AMARAI, Les Dossiers du Centre Régional d'Archéologie d'Alet, $n^{\circ} \mathrm{AE}, 144 \mathrm{p}$.

DAIRE M.-Y., LANGOUËT L., 2008b. Sur la chronologie des pêcheries fixes ; le point de vue de l'archéologue, dans DAIRE M.-Y. et LANGOUËT L. (dir.), Les pêcheries de Bretagne, Archéologie et Histoire des pêcheries d'estran, Coéd. CeRAA/AMARAI, Les Dossiers du Centre Régional d'Archéologie d'Alet., $\mathrm{n}^{\circ} \mathrm{AE}, \mathrm{p}$. 51-60.

DAIRE M.-Y., LANGOUËT L., 2010. Les anciens pièges à poissons des côtes de Bretagne, un patrimoine au rythme des marées..., Coédition CeRAA/AMARAI, Les Dossiers du Centre Régional d'Archéologie d'Alet, $\mathrm{n}^{\circ} \mathrm{AG}, 165 \mathrm{p}$.

DAIRE M.-Y., MARGUERIE D., FILY M., BAUDRY A., QUESNEL L., 2011. Le plateau littoral du bas-Léon au $\mathrm{I}^{\mathrm{er}}$ millénaire av. J.-C. : Regards croisés sur les dynamiques du peuplement et du paysage, Norois, ce volume, p. 95-119.

FISCHER A., 2007. Costal fishing in Stone Age Denmark, dans MILNER N. et al. (dir.), Shell Middens in Atlantic Europe, Oxbow Books, 336 p.

GIOT P.-R., 1990. Le niveau de la mer : changeant, fluctuant, mouvant..., Bulletin de l'AMARAI, $\mathrm{n}^{\circ} 3$, p. 5-16.

GIOT P.-R., MONNIER J.-L., L'HELGOUAC'H J., 1998. Préhistoire de la Bretagne, Rennes, Ouest-France Université, $589 \mathrm{p}$.

KLEIN J., LERMAN J.-C., DAMON P. E., RALPH E. K., 1982. Calibration of radiocarbon dates, Radiocarbon, V, 24, p. 103 sq. 
LANGOUËT L., DAIRE M.-Y., 2009. Ancient maritime fish-traps of Brittany (France) : a reappraisal of the relationship between human and coastal environnement during the Holocene, Journal of Maritime Archaeology, Springer, vol. 4-2, p. 131-148.

LANGOUËT L., DAIRE M.-Y., 2010. Méthodologie de caractérisation des anciennes pêcheries d'estran, Les Dossiers du Centre Régional d'Archéologie d'Alet, n 38, p. 59-62.

LANGOUËT L., GOULPEAU L., 1980. Fouille d'un foyer de briquetages à l'Île à Poule, en Kerbors (Côtesd'Armor), Les Dossiers du CeRAA, $\mathrm{n}^{\circ}$ 8, p. 99-106.

LANGOUËT L., LE QUELLEC J.-M., PRIGENT G., 2008. Des pêcheries monastiques insulaires en Bretagne, Bulletin de l'AMARAI, $\mathrm{n}^{\circ} 21$, p. 59-80.

LARGE J.-M., BIROCHEAU P., CORSON S., COUSSEAU F., LARGE C., TORTUYAUX J.-P., 2009. Une archéologie des pêcheries d'estran : l'anse aux Moines et la pointe du Vieux Moulin au Château-d'Olonne, en Vendée, Groupe Vendéen d'Études Préhistoriques, ${ }^{\circ}$ 45, p. 4-45.

LEVASSEUR 0., 2008. Les pêcheries sur les côtes septentrionales de Bretagne d'après les procèsverbaux d'inspection de François Le Masson du Parc, dans DAIRE M.-Y., LANGOUËT L. (dir.), Les pêcheries de Bretagne, Archéologie et Histoire des pêcheries d'estran, Coédition CeRAA/AMARAI, Les Dossiers du CeRAA, n AE, p. 11-33.

LÜBKE H., 2007. Traces of a submerged world - Investigations on submarine stone age sites, on the South Western Baltic of Mecklenburg-Vorpomern, Germany, Abstract book of the $13^{\text {th }}$ International Congress of the EAA (European Association of Archaeologists), Zadar (Croatia), p. 203-204.

MAARLEVELD T. J., 1993. Between frugality and eclectism. Some thoughts on the management of an archaeological cornucopia. (Bulletin of the Autralasian Institute for Maritime Archaeology, publication électonique [http://www.aav.J.-C..se/ ppa/publ/aima172.htm].

MCQUADE M., O'DONNELL L., 2007. Late Mesolithic fish traps from the Liffey estuary, Dublin, Ireland. Antiquiyt, vol. 81, $\mathrm{n}^{\circ} 313$, p. 569-584.

MAZÉAS M., 2008-2009. Les établissements romains de Plomarc'h, à Douarnenez : salaisons et garum, Bulletin de la Société d'Archéologie et d'Histoire du Pays de Lorient, vol. 37, p. 33-50.

MORZADEC-KERFOURN M.-Th., 1969. Variations de la ligne de rivage au cours du post-glaciaire le long de la côte nord du Finistère. Analyses polliniques de tourbes et de dépôts organiques littoraux, Bulletin de l'AFEQ, 4, p. 285-317.

-, 1974. Variations de la ligne de rivage armoricain au Quaternaire. Analyses polliniques de dépôts organiques littoraux, Mémoire de la Société Géologique et Minéralogique de Bretagne, 17, 208 p. O'SULLIVAN A., 1994. Harvesting the Waters, Archaeology Ireland, vol. 8-1, n² 27, p. 10-12.

,- 2001 . Foragers, farmers, and fishers in the coastal landscape : an intertidal archaeological survey of the Shannon estuary, Dublin, Royal Irish academy, 345 p.

,- 2003. Place, memory and identity among estuarine fishing communities: interpreting the archaeology of early medieval fish weirs, World Archaeology, vol., n 3, p. 449-468.

PEDERSEN L., 1995. 7000 years of fishing ; stationary fishing structures in the Mesolithic and afterwards, in Fisher A. (ed.), Man and sea in the Mesolithic, Oxford, p. 75-86

PIRAZZOLI P. A., 1991. World atlas of Holocene sea-level changes, vol. 58, London, Elsevier, coll. « Elsevier Oceanography Series », $300 \mathrm{p}$. 
PONDAVEN P., 1992. De quelques toponymes révélateurs de la présence de pêcheries anciennes sur le littoral du Bas-Léon, Bulletin de l'AMARAI, nº 5, p. 57-65.

SHENNAN I. (ed.), 1989. Late Quaternary, sea-level changes and crustal movements in the British Island, Journal of Quaternary Science, vol. 4, $\mathrm{n}^{\circ}$ 1, p. 1-90.

STÉPHAN P., 2011. Colmatage sédimentaire des marais maritimes et variations relatives du niveau marin au cours des 6000 dernières années en rade de Brest (finistère), Norois, ce volume, p. 9-37.

\section{NOTES}

1. Pour la Basse-Normandie, nous renvoyons aux travaux coordonnés pas C. Billard et V. Bernard autour des installations de Saint-Jean-le-Thomas, du Havre de Portbail, etc. (Billard et al., 2006) et pour les Pays de la Loire, aux recherches fédérées par J.-M. Large autour du Groupe Vendéen d'Études Préhistoriques (Large et al., 2009). Pour la Bretagne, le programme pêcheries est coordonné par M.Y. Daire et L. Langouët et placé sous l'égide de l'Association pour la Recherche Archéologique dans les îles (AMARAI) et du Centre de Recherches en Archéologie, Archéosciences et Histoire (CReAAH, UMR 6566).

2. Dans le cadre du programme breton, la réalisation de la base de données PEB (Pêcheries d'Estran de Bretagne) a permis de localiser et caractériser plus de 720 anciens barrages à poissons), entre le Mont-Saint-Michel et l'embouchure de la Vilaine. Une vingtaine de variables est associée à chacune des pêcheries au sein de la base de données PEB (avec, entre autres, la commune littorale, la dénomination locale, la toponymie marine, les coordonnées Lambert II étendu, le niveau 0 SHOM, le marnage maximal du site, la documentation, les photographies au sol et aériennes, la nature des vestiges, la typologie, les dimensions, les éléments de datation... Une trentaine de prospecteurs, chercheurs et informateurs participent à l'étude des pêcheries en Bretagne.

3. En Bretagne, les marées, dues à la combinaison des attractions gravitationnelles du soleil et de la lune, sont de type semi-diurne. La terre fait une rotation complète sur elle-même en 24 heures tandis que la lune tourne autour de la terre avec une périodicité de 24 h 50'. Cette différence de périodes entraîne qu'il n'y a qu'environ 700 marées par an.

4. Cartes 99-774 et 99-775 des ingénieurs-géographes militaires, Service historique de la Défense, département de l'Armée de terre, J10C 289, Vincennes.

5. Des tests de datations par la méthode OSL (Optically Stimulated Luminescence) sont en projet sur certains vestiges de Bretagne.

6. Le recensement par F. Le Masson Du Parc est lié à l'application des ordonnances royales exigeant la destruction de tous les barrages de pêcheries construits après 1544 . 


\section{RÉSUMÉS}

En conséquence directe du récent développement de l'archéologie des anciennes pêcheries, dans l'Ouest de la France (Daire et Langouët, 2008, 2010 ; Langouët et Daire, 2009) une réflexion s'engage sur l'évolution chronologique de cette forme d'exploitation du littoral au regard des variations des niveaux marins. À partir de l'analyse des règles ayant conditionné l'installation de barrages, une approche théorique très novatrice est proposée puis discutée à partir d'une zone test: le Bas-Léon (Finistère). Le choix de ce secteur géographique est justifié par l'abondance relative de structures de barrages (72) géoréférencées (y compris par rapport au niveau zéro des cartes marines) et par l'existence de données topographiques et chronologiques (prélèvements et datations) qui, bien qu'anciennes et nécessitant une actualisation, fournissent un schéma des variations relatives du niveau marin depuis sept millénaires, sur ce secteur géographique.

As a direct consequence of recent development in ancient fish-traps archaeology, in Western France, (Daire et Langouët, 2008, 2010; Langouët et Daire, 2009), a discussion recently emerged about the chronological evolution of this form of coastal resources exploitation, as regards its links with sea level changes. From the analysis of rules having conditioned the installation of the weirs, a very innovative theoretical approach is proposed here and then discussed from a test zone: the Bas-Léon (Finistère). This area has been selected on the basis of several criteria: the relative abundance of fish weirs structures (72), the precisions of their location (including by reference to the present sea level reference), and the existence of topographic and chronological data (samplings and dating) related to these archaeological structures. Even though it appears necessary to update some ancient observations, this data can provide a solid basis for new considerations on sea level changes, for seven thousands of years, in this geographic area.

\section{INDEX}

Mots-clés : pêche, barrage à poissons, niveau marin

Keywords : traps, fishing, weirs, sea level

\section{AUTEURS}

\section{MARIE-YVANE DAIRE}

Centre de Recherche en Archéologie, Archéosciences, Histoire, UMR 6566 (Université de Rennes 1), Bâtiment 24-25, campus de Beaulieu - 35042 Rennes Cedex, France marieyvane.daire@univ-rennes1.fr

\section{LOÏC LANGOUËT}

Association Manche Atlantique pour la Recherche Archéologique dans les îles (Université de Rennes 1), Résidence Parc Saint-Laurent, 1 rue de la Tauvrais - 35700 Rennes, France Langouet.loic@wanadoo.fr 